\title{
Analyser une transition agro-alimentaire par les flux d'azote : Aussois un cas d'étude du découplage progressif de la production et de la consommation
}

\section{Analyzing a food system transition by nitrogen flow: Aussois a case study of the progressive decoupling of production and consumption}

Thierry BONAUDO,

UMR SADAPT, AgroParisTech, INRA, Université Paris-Saclay, 75005, Paris, France thierry.bonaudo@agroparistech.fr

Auteur correspondant

Gilles BILLEN,

CNRS et Université Pierre et Marie Curie, laboratoire Metis, Paris

Josette GARNIER,

CNRS et Université Pierre et Marie Curie, laboratoire Metis, Paris

Fabienne BARATAUD,

Inra, Unité ASTER (Agro-Systèmes, Territoires, Ressources), Mirecourt

Sabine BOGNON,

INSHS (Institut des sciences humaines et sociales), UMR 8504 Géographie-cités, Paris

Pauline MARTY,

INSHS (Institut des sciences humaines et sociales), UMR 8504 Géographie-cités, Paris

Denis DUPRE

Université de Grenoble, laboratoires CERAG et INRIA (STEEP), Grenoble

\section{Mots-clés}

Ecologie territoriale, système agro-alimentaire, élevage, transition, Aussois,

\section{Keywords}

Territorial ecology, agro-food system, farming, transition, Aussois 
Résumé

Nous reconstituons l'évolution du système agro-alimentaire de la commune d'Aussois, en considérant les flux d'azote et de monnaie au cours du $20^{\text {ème }}$ siècle. Deux périodes sont distinguées : de 1925 à 1965, la période est caractérisée par des systèmes agro-pastoraux vivriers et autonomes, tandis que la seconde période de 1965 à nos jours, se définit par des systèmes pastoraux spécialisés et plus intensifs avec une autonomie de 67\%. L'efficacité d'utilisation de l'azote, définie comme la proportion d'azote des intrants se retrouvant dans les produits sortant, n'évolue presque pas entre les deux périodes. Seul 10\% de l'azote des intrants est fixé dans les produits sur la première période et $11 \%$ pour la deuxième période. La productivité, soit la quantité de bien produit par surface, est multipliée par 2,5 pour atteindre aujourd'hui 7,2 $\mathrm{kg}$ $\mathrm{N} / \mathrm{ha}$ /an. Enfin, on remarque une évolution vers une disjonction des productions communales et de la demande alimentaire locale qui s'approvisionne aujourd'hui massivement sur le marché national. Cette insertion dans une dynamique nationale entraine une ouverture du cycle d'azote et le passage d'une situation de flux monétaires faibles, intimement liés aux flux d'azote, à des flux monétaires importants et indépendants des flux d'azote.

\section{Abstract}

We reconstructed the one-century evolution of the agri-food system of the municipality of Aussois. We considered the nitrogen and money flows. Two periods are distinguished: from 1925 to 1965, characterized by autonomous agro-pastoral systems, while the second period from 1965 to the present, is defined by specialized and intensive grazing systems with an autonomy of $67 \%$. Nitrogen use efficiency, defined as the proportion of nitrogen inputs fixed in post products, almost no changes between the two periods. Only $10 \%$ of nitrogen inputs is fixed in the products of the first period and $11 \%$ for the second period. Productivity, or the amount of products produced by surface is multiplied by 2.5 today to $7.2 \mathrm{~kg} \mathrm{~N} /$ ha / year. Finally, there is a disjunction of local production and the food demand linked to the national market. This insertion of the territory in a national dynamics causes an opening of the nitrogen cycle and an economic transition from a low cash flow closely linked to nitrogen flow, to a significant cash flows independent of nitrogen flow. 


\section{$-1-$ \\ Introduction}

Un aspect fondamental du fonctionnement d'un territoire est la manière dont ses habitants l'exploitent et le transforment pour y produire les moyens de leur subsistance, en particulier alimentaire. Il existe de nombreuses études mettant en évidence la transformation des systèmes agraires au cours du $20^{\text {ème }}$ siècle, leur intensification et leur spécialisation (MALASSIS, 1994 ; Bourgeois et DEMOTES-MAINARD, 2000 ; MAZOYER et RoudART, 2002). Mac DonALD et al., 2000 montrent en particulier l'évolution des systèmes de production et des paysages en zone de montagne. L'originalité de notre article est de quantifier et objectiver le fonctionnement d'un système agro-alimentaire dans son ensemble par l'étude des flux de matière. En effet, au-delà de la production agricole, et de son corolaire le maintien sur le long terme de la fertilité des sols, c'est l'ensemble du système agro-alimentaire qui structure les territoires. Nous définissons le « système agro-alimentaire », comme l'articulation de l'ensemble des activités et services liés à la production, la transformation, la commercialisation et la consommation alimentaire (MALASSIS, 1994). Cette acception large articule production et demande alimentaire, et inclut un grand nombre d'acteurs et de territoires en interactions. C'est cet objet d'étude complexe que nous analysons.

Pour cela, nous mobilisons la méthode du métabolisme territorial, pour quantifier les flux et stocks de matières d'un système alimentaire d'une petite commune de la vallée de Maurienne en Savoie : Aussois. Cet inventaire, situé dans l'espace et le temps, permet d'appréhender la réalité biogéochimique du fonctionnement des territoires et d'analyser conjointement des processus naturels et techniques (BARLES, 2010, BILLEN et al., 2014). Le métabolisme du système alimentaire des villes et sa gouvernance sont de mieux en mieux documentés (voir REG + petros), ainsi que la mutation des relations entre la société et son milieu sur des temps plus ou moins longs (FISCHER-KOWALSKI ET HABERL, 2007). Notre ambition est d'analyser l'évolution dans le temps du métabolisme du système agro-alimentaire d'un village de montagne, et de contribuer à une meilleure connaissance de ces mutations.

\section{$-2-$ \\ Cadre théorique et méthode}

Notre ambition étant de quantifier l'évolution du système alimentaire d'Aussois, nous avons identifié deux périodes représentatives de systèmes agro-alimentaires distincts. La première période de 1925 à 1965, est caractérisée par une activité agricole dominante, composée de systèmes agro-pastoraux diversifiés et autonomes. La deuxième période de 1965 à nos jours, est caractérisée par le désenclavement de la commune, la construction de barrages hydroélectriques et la montée en puissance des activités de tourisme de neige. L'activité agricole est marquée par la constitution de grands systèmes pastoraux spécialisés et intensifiés. Pour chaque période, nous avons reconstitué un "système de production exemple », qui est une représentation cohérente et crédible du fonctionnement technico-économique moyen d'une exploitation agricole. Cependant l'ensemble des caractéristiques du système exemple ne se retrouve ni parfaitement, ni entièrement dans des exploitations réelles; en ce sens, ces systèmes exemples se rapprochent de la définition «d'idéaux types » de Max Weber. Chaque système exemple a été construit par itération entre bibliographie sur les systèmes agricoles de Maurienne, statistiques agricoles et entretiens permettant d'identifier les principaux critères structuraux et fonctionnels. Nous avons mobilisé: 
- Des études, notamment de la géographie rurale et des sociétés d'histoire locale ou rapports technico-économiques décrivant l'utilisation agricole et pastorale des sols, les calendriers fourragers, les itinéraires techniques, le calendrier et les lieux (foires) des ventes des produits des exploitations (BOZON, 1986, ONDE, 1932 et 1937; GARDELLE, 1965, 1976 ; Guerin, 1972 ; GALlety, 1983 ; Viallet, 1993 ; INAO, 2010 ; IDELe, 2010 ; AGRESTE, 2011). Le musée agro-pastoral d'Aussois reconstituant la vie rurale avant les années 60 a été aussi source d'information sur les pratiques agricoles de la $1^{\text {ère }}$ période d'étude, de même qu'un entretien avec un groupe de personnes âgées sur les pratiques alimentaires et commerciales au village pendant leur enfance.

- Les statistiques agricoles de 1955 (RGA, 1955, in RAMBAUD et VINCIENNE, 1964). et de 2010 concernant le nombre d'exploitations agricoles, leurs surfaces, les cheptels convertis en Unité Gros Bovin (UGB), les productions animales (lait, viande, œuf) et les productions végétales (prairies, fourrages, céréales, pommes de terre, oléo-protéagineux, ...) à l'échelle de la commune. Ces statistiques en particulier ont été rapportées au nombre moyen d'exploitations aussoyennes au cours de la période 1925 - 1965 pour la reconstitution du système agraire exemple.

- Huit entretiens d'agriculteurs et, d'experts agricoles de la région.

Une fois les systèmes agraires exemples reconstitués et les flux de matières brutes évalués (intrants, produits et coproduits et effluents), nous avons converti tous les flux en une seule unité pour pouvoir les comparer et les agréger. Comme pour la plupart des études en la matière, nous avons choisi l'azote. En effet, l'azote est un bon indicateur du fonctionnement des systèmes agro-alimentaires, c'est un élément fondamental de la fertilité des sols et de la croissance des plantes. En tant que constituant essentiel des protéines, il est aussi fondamental pour l'alimentation humaine et animale. L'azote permet d'appréhender conjointement les productions végétales et animales, mais aussi les apports d'intrants sous forme d'engrais (minéraux et organiques), d'alimentation animale importée, ou encore la fixation atmosphérique d'azote (BILlEN et al., 2014, BODIRSKY et al., 2014). Les coefficients d'azote de chaque matière brute sont basés sur la bibliographie (LASSALETTA et al., 2014 a-b ; BILLEN et al., 2014, 2015). Nous ne considérons que la fixation d'azote atmosphérique par les prairies naturelles, en nous basant sur une étude de ANGLADE et al., 2015. Les effluents ont été calculés à partir des estimations d'UGB, en reprenant un coefficient d'excrétion moyen de $85 \mathrm{~kg}$ N/UGB/an (CORPEN 1999, 2001). La taille des animaux (poids équivalent carcasse) étant nettement plus réduite pour la $1^{\text {ère }}$ période, nous avons diminué de 40\% le rapport UGB/tête actuel ( $c f$. Tableau 1). Enfin, la cohérence générale du système respecte la loi de conservation de masse de Lavoisier : "Rien ne se perd, rien ne se crée, tout se transforme », soit Flux Intrants - Flux Produits + Variation de stock $=0$. Nous mobilisons la méthode de représentation GRAF (Generic Representation of Agro-Food Systems) pour formaliser l'ensemble de ces éléments en fonction de l'utilisation des sols (BILLEN et al., 2014, 2015). 
Tableau 1. Principaux coefficients utilisés

\begin{tabular}{|l|c|}
\hline \multicolumn{2}{|c|}{$\begin{array}{c}\text { \% d'azote dans les } \\
\text { produits }\end{array}$} \\
\hline Produit & \% Azote \\
\hline Céréales & 1,8 \\
\hline Pommes de terre & 0,26 \\
\hline Foin sec & 1,6 \\
\hline Lait & 0,53 \\
\hline Viande & 3,1 \\
\hline Euf & 1,9 \\
\hline
\end{tabular}

\begin{tabular}{|l|c|c|}
\hline \multicolumn{3}{|c|}{$\begin{array}{c}\text { Équivalent en UGB par tête animale } \\
\text { (1 UGB excrète } 85 \mathrm{kgN} / \mathrm{an})\end{array}$} \\
\hline \multicolumn{1}{|c|}{ Espèce } & $1^{\text {ere }}$ période & $2^{\text {ème }}$ période \\
\hline Vache laitière & 0,54 & 0,9 \\
\hline Veau $<1$ an & 0,12 & 0,2 \\
\hline Génisse 1-2 ans & 0,36 & 0,6 \\
\hline Génisse 2-3 ans & 0,48 & 0,8 \\
\hline Brebis & 0,09 & 0,15 \\
\hline Agneau & 0,03 & 0,05 \\
\hline Chèvre & 0,1 & 0,17 \\
\hline Cochon & 0,16 & - \\
\hline Poulet & 0,005 & - \\
\hline Lapin & 0,03 & - \\
\hline Mulet & 1 & - \\
\hline
\end{tabular}

En quantifiant avec la même unité les intrants, la production de biens et de déchets cette méthode permet de calculer différents indicateurs de performance : i) indicateur de production animale et végétale en $\mathrm{kg}$ d'azote par ha et par an ii) indicateur d'azote apporté aux sols en $\mathrm{kg}$ d'azote par ha et par an, iii) indicateur d'efficacité d'utilisation de l'azote à l'échelle de l'exploitation correspondant à l'ensemble de l'azote entrant (fertilisation organique et minérale, fixation symbiotique d'azote, alimentation du bétail importé) divisé par l'azote fixé dans les produits animaux et végétaux consommés par l'homme.

En parallèle, nous avons reconstruit un régime alimentaire type pour chaque période, complété par une estimation de la population totale de la commune incluant les flux de touristes pour la période contemporaine. Pour ce faire, en complément des données bibliographiques (GARDELLE, 1965 ; JAIL, 1969 ; GUERIN, 1972 ; MARNEZY, 1979), une dizaine d'entretiens ont été réalisés avec différents acteurs du système alimentaire incluant la distribution (deux superettes, un primeur) et la consommation (cinq habitants du village, deux agriculteurs), deux acteurs de la restauration collective et privée.

Une des originalités de ce travail est de développer conjointement une analyse des flux de matières et des flux monétaires appréhendés comme un moyen de gérer les flux de matières dans le temps et dans l'espace.

\section{$-3-$ \\ Un siècle à Aussois}

La transformation radicale du système agro-alimentaire de la commune d'Aussois (vallée de la Haute Maurienne en Savoie) au cours du XXème siècle apparaît manifeste au simple examen des données statistiques relatives à la population et au nombre d'exploitations agricoles de la commune ( $c f$. Figure 1). Durant la période de 1925 à 1960 précédant les grands travaux hydroélectriques et l'aménagement de la station de ski, Aussois compte de 350 à 500 habitants (sur un territoire de $42,6 \mathrm{~km}^{2}$ ) et une soixantaine d'exploitations agricoles. Autant dire que toute la population est concernée par l'agriculture. La taille moyenne des exploitations est d'une dizaine d'hectares (hors estives), avec moins d'un quart de terres arables destinées à la culture de céréales. Même si l'élevage est dominant, avec 0,4 UGB/ha (hors estives), la densité animale est faible (RGA, 1955, in RAMBAUD et VINCIENNE, 1964).

Aujourd'hui, la commune compte environ 650 habitants (INSEE, population légale 2013), et nous estimons que l'accueil des vacanciers équivaut à 1800 habitants permanents 
supplémentaires. Les exploitations agricoles au nombre de 6, utilisent à peu près la même surface de SAU que pour la première période (400 ha, hors estives). L'agriculture de la commune a suivi une dynamique générale en France de concentration foncière, d'intensification et de spécialisation (BOURGEOIS et DEMOTES-MAINARD, 2000). La culture de céréales sur les terres arables a pratiquement disparu et les exploitations se sont spécialisées sur l'élevage bovin laitier et ovin ( $c f$. Figure 1). On compte aujourd'hui une densité animale moyenne supérieure à $0,5 \mathrm{UGB} / \mathrm{ha}$ (hors estives).

Figure 1. Évolution de la population, du nombre d'exploitations agricoles, de la surface agricole et du cheptel dans la commune d'Aussois depuis le début du XXème siècle

\section{Population}

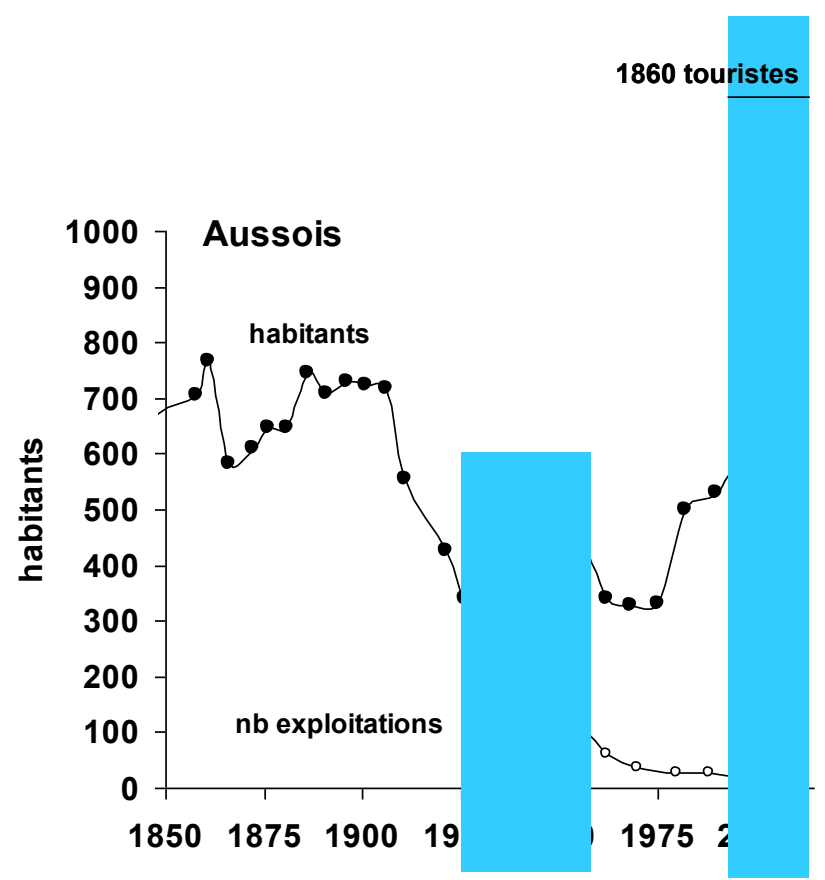

\section{Surf. agricole et cheptel}

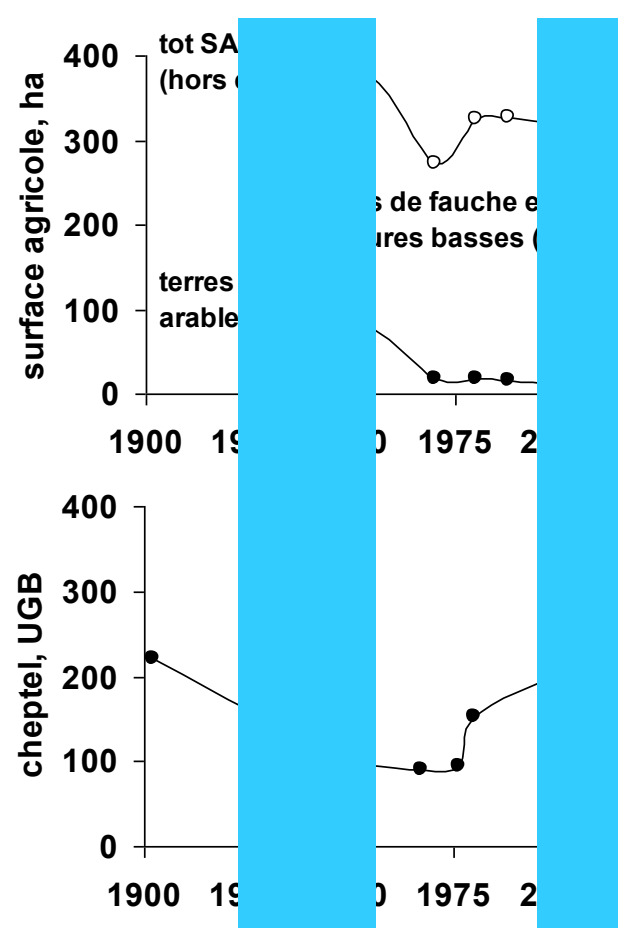

Sources : RGA 1955, in RAMBAUD et VINCIENNE, 1964 ; RA 2010 ; INSEE, population légale 2011 


\section{La période 1925-1965 : une agriculture agro-pastorale vivrière}

\subsection{Fonctionnement général d'un système agro-pastoral vivrier}

Notre système exemple est composé d'une famille d'une dizaine de personnes sur trois générations, exploitant une SAU de 22 ha. L'essentiel des surfaces sont composées de «prés bas » $(10$ ha $)$ et de « prés hauts » (10 ha) pâturés et fauchés. Du fait du climat montagnard, les prés hauts ou alpages ne sont exploités qu'en été (juin à septembre) à partir d'une habitation temporaire. En plus des pâturages, la famille exploite dans la vallée 0,8 ha de terres arables, assolées en seigle, orge, avoine et pomme de terre. Enfin, les familles ont un potager d'environ $500 \mathrm{~m}^{2}$ pour leur consommation (cf. Figure 2).

Le troupeau diversifié est composé de quelques têtes de bovins généralement de race Tarine (plus rarement d'Abondance), de quelques chèvres laitières et de moutons pour fournir de la laine et de la viande. Ces espèces avec des tailles, des aptitudes physiologiques et des comportements alimentaires différents utilisent des ressources fourragères complémentaires et fournissent des produits diversifiés. Caprins et ovins exploitent des zones d'alpage plus accidentées que les bovins. L'ensemble des animaux sont robustes, ils supportent à la fois une claustration longue en hiver et ils développent des aptitudes à la marche en alpages. Les ressources fourragères abondantes au printemps et en été permettent un pic de production de lait, qui est transformé en fromage de garde sur les alpages l'été et l'hiver à la maison ou à la fruitière du village. Des animaux de basse-cour accompagnent aussi la famille en alpages, il y a des poules et des lapins ainsi que deux à trois porcelets qui sont engraissés à partir des résidus au premier rang desquels le petit lait issu de la transformation fromagère. Enfin, la famille possède un mulet, animal de travail essentiel à la ferme. Il permet de monter le matériel nécessaire à la vie en alpage mais aussi de descendre les fromages et une partie du foin essentiel à l'alimentation du bétail en l'hiver. Cet animal est au centre du transfert de fertilité des alpages vers la vallée (prés bas, terres arables, potager). Le mulet permet aussi le transport du fumier et le labour des terres arables. Pour les familles les plus modestes, le mulet est parfois partagé. Sans ces capacités de trait, il n'y a pas ou peu de transport des éléments fertilisants et pas d'autonomie en azote de l'exploitation.

Cette diversité biologique permet à l'élevage de remplir plusieurs fonctions essentielles : une fonction alimentaire avec l'autoconsommation de viande et de lait tout au long de l'année, une fonction de trésorerie avec la vente d'animaux et de produits, une fonction de capitalisation à moyen long terme, une fonction de travail et de transfert de fertilité primordiale au maintien de la fertilité des terres arables...

\subsection{Flux d'azote d'un système agro-pastoral vivrier}

La figure 2 illustre les flux d'azote liés aux pratiques agricoles décrites ci-dessus. Cette figure illustre le rôle central des animaux dans la sécurité alimentaire de la famille et dans le maintien de la fertilité des sols par leur capacité à restituer par leurs fèces de l'azote sous forme assimilable par les plantes.

Les flux d'azotes sont relativement réduits et localisés. Les productions animales sont de $2,8 \mathrm{~kg} \mathrm{~N} / \mathrm{ha} /$ an et les productions végétales consommées par l'homme de $0,5 \mathrm{~kg} \mathrm{~N} / \mathrm{ha} / \mathrm{an}$. Les entrées totales d'azote dans le système agricole sont de $33 \mathrm{~kg} \mathrm{~N} / \mathrm{ha} /$ an (fertilisation organique et fixation symbiotique). Les seules entrées d'azote dans le système agro-alimentaire sont la fixation d'azote par les légumineuses des prairies ainsi qu'une faible importation d'azote via l'alimentation humaine $(1,5 \mathrm{~kg} \mathrm{~N} / \mathrm{an}$ soit $3 \%$ de la consommation humaine). Les sorties d'azote sont les exportations de produits animaux $(1,3 \mathrm{~kg} \mathrm{~N} / \mathrm{an}$ de viande et fromage). L'efficacité d'utilisation de l'azote à l'échelle de la ferme est de 10\% (ensemble de l'azote entrant comme fertilisation organique et fixation symbiotique d'azote divisé par l'azote fixé dans les produits animaux et végétaux consommé par l'homme), c'est un chiffre cohérent avec ceux de la littérature de systèmes extensifs utilisant peu ou pas de fertilisant (BILLEN et al., 2014). 
Les prés hauts sont indispensables à l'alimentation du bétail par le pâturage en été mais aussi par les coupes de foin, base de l'alimentation en hiver. On estime que $30 \%$ du foin provient des alpages. Ce transfert d'azote des alpages vers la vallée via le foin puis le fumier accumulé en étable durant l'hiver est essentiel au maintien de la fertilité des terres arables, du potager et des prés bas. Les terres arables sont destinées principalement à l'alimentation du mulet (plus de $60 \%)$ et humaine $(25 \%)$.

Dans ce contexte montagnard avec des ressources limitées, l'élevage est au cœur de la production agricole et de la sécurité alimentaire, soit directement par l'autoconsommation de produits animaux, soit indirectement par le maintien de la fertilité des terres arables et du potager. La diversité des animaux (ruminants et monogastriques) permet de valoriser l'ensemble des ressources alimentaires locales (fourrage, résidus ménagers et issus de la transformation fromagère...). Sur $73 \mathrm{~kg}$ d'azote produits par an pour l'alimentation humaine (62 kg d'origine animale et $11 \mathrm{~kg}$ d'origine végétale) près de $65 \%$ sont autoconsommés et moins de $35 \%$ sont vendus. Les ventes concernent uniquement des produits animaux, principalement vache de réforme et fromage. Seul 3\% de l'azote du régime alimentaire est importé (pâtes, riz, polenta, farine de châtaignes, fruits et légumes secs, ...). Ce système agro-pastoral vivrier conduit à un régime alimentaire riche en protéines animales, avec $3,5 \mathrm{~kg} \mathrm{~N}$ animal/an/personne contre 1,25 $\mathrm{kg} \mathrm{N}$ végétal/an/personne (plus de $70 \%$ de l'azote est d'origine animale : viandes, charcuteries, fromages par exemple).

Figure 2. Les flux d'azote en kg/an d'un système agro-pastoral vivrier exemple (1925-1960)

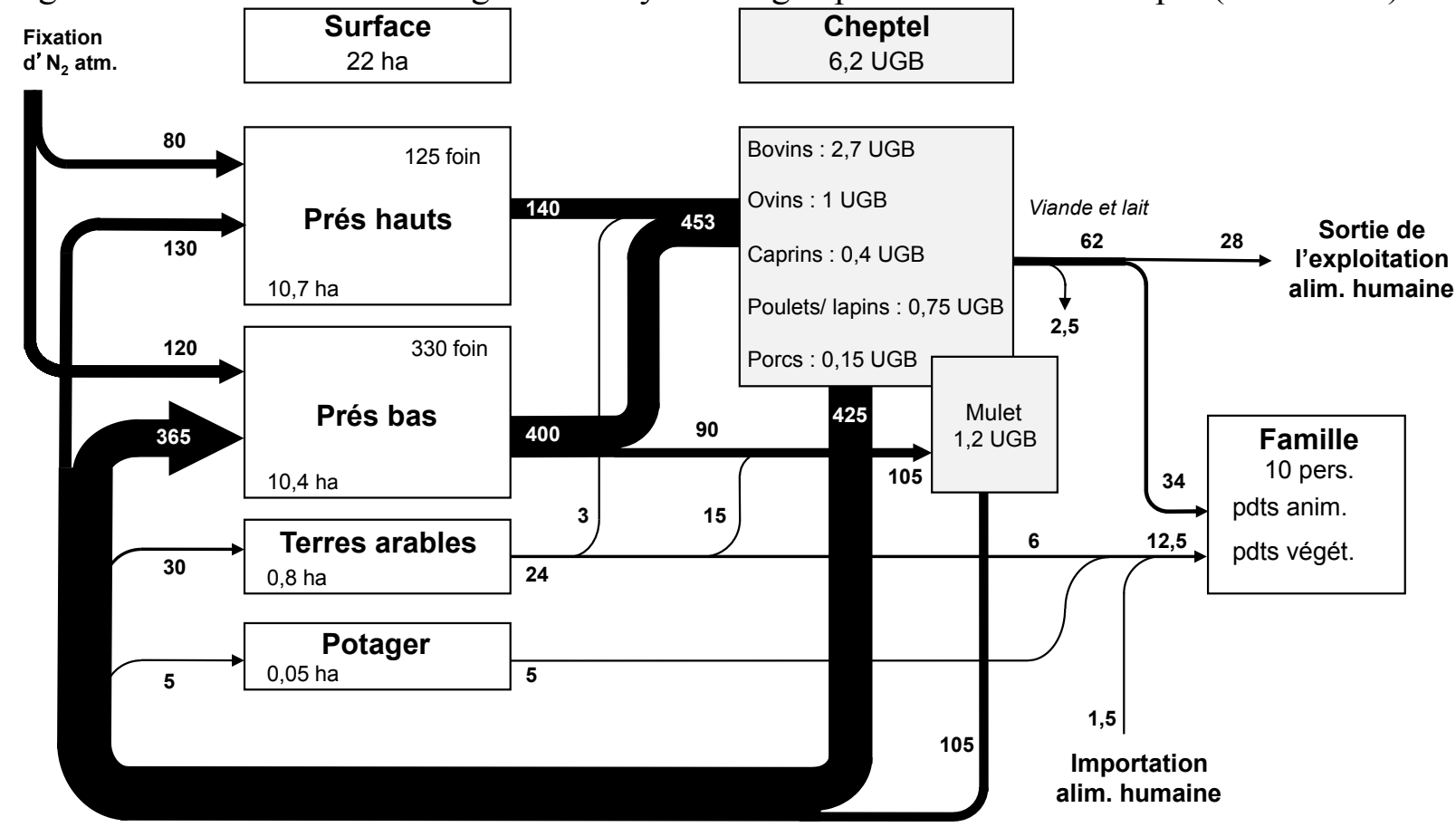

A l'échelle du ménage agricole, les productions animales sont essentielles à l'accès à la monnaie. Les fromages de gardes produits dans les alpages l'été peuvent être revendus aux foires de la vallée grandes tailles à pâte cuite pressée se conservent longtemps et sont faciles à transporter. Ils sont vendus et expédiés vers les pôles urbains (Turin, Lyon ou plus éloignés comme Paris). S'ajoute à ces entrées fiduciaires la vente ponctuelle des vaches de réformes ou des jeunes bestiaux surnuméraires dans les foires de la vallée. 


\subsection{Flux monétaires d'un système agro-pastorale vivier}

De nombreux documents s'intéressant à la Maurienne des XVIIème et XVIIIème siècle témoignent d'une pénétration de la monnaie dans l'économie agro-pastorale de la vallée, marquée cependant par sa faiblesse. Une partie des échanges nécessaires au maintien du système agro-pastoral d'Aussois se font au sein de la vallée, qui outre le vin fournit une partie des matériaux de construction (bois, chaume, pierre et plus rarement ardoise) (Barles ???). Il est à noter que dans le cadre du village ou de la vallée, certains échanges n'impliquent pas toujours le fiduciaire : par exemple les vignes de moyenne et basse Maurienne appartiennent en partie à la haute Maurienne (Rambaud 1964). Le sel des Salines de Moutiers, situées dans la vallée voisine de la Tarentaise, fait l'objet d'une contrebande active vers l'Italie, et peut faire office de substitut à la monnaie en fiduciaire pour l'échange - il a une place importante dans les dons entre familles du village (Arbos 1919).

Mais la Maurienne ne fonctionne pas en autarcie complète, elle insérée dans des réseaux d'échanges qui sont une source de monnaie directe et indirecte pour la société agro-pastorale. Ceux-ci se développent notamment au XVIIIème siècle. En premier lieu, la vente des produits agricoles : la vallée participe à l'approvisionnement en jeunes bestiaux, bestiaux engraissés et mulets des marchés de Suze et de Turin via les foires de la vallée (Grillet J.-L., op. cit., vol. 3, p. 12. ; Arbos, 1919). Ensuite, la situation géographique de la Maurienne, route principale entre la France et l'Italie, via le col du Galibier puis via le col du Mont-Cenis à partir de 1830 (Chamussy 1968), est un élément important de la pénétration de la monnaie dans l'économie de la vallée. Dès avant la route carrossable du col du Mont-Cenis, ouverte en 1813, on note l'activité intense des muletiers, porteurs et aubergistes (Palluel J., op. cit., 3e partie, p. 185-186). La route devenue carrossable, le trafic de voitures de marchandises et mulets de charge explose (Cimaz, 1997). Le développement des fonctions commerciales et de transit liées aux échanges transfrontaliers et les besoins de main d'œuvre et matériaux pour la construction des routes et la maintenance des voitures irriguent la vallée d'une nouvelle activité économique. L'ouverture vers l'Italie amène une consommation courante de denrées non produites dans l'exploitation (riz, pâtes, polenta, chaussures et outillages ramenés du marché de Turin (Cimaz, 1997)), tandis que le nombre de foires dans la vallée et les cheptels commercialisés augmentent (rambaud vincienne 1964). L'ouverture du tunnel ferroviaire du Fréjus dans les années 1870 (Cimaz, 1997) qui dévie le trafic vers l'Italie en aval, viendra mettre un coup d'arrêt aux activités de transit, laissant la Haute Maurienne retourner au statut de « fin de vallée » jusqu'à l'arrivée de la voiture personnelle (Chamussy, 1968).

L'effet de ces vecteurs de pénétration monétaire de l'économie de la vallée est localement variable, et Aussois est resté en partie en retrait du mouvement général. Une fois la route du Montcenis carrossable, on note une croissance importante des fonctions commerciales liées au transit, mais seulement dans les bourgs-étapes et ceux qui accueillent les maisons de roulage (Cimaz, 1997), or Aussois est à $20 \mathrm{~km}$ du bourg-étape le plus proche. Les flux monétaires issus de la vente des produits animaux sont plutôt faibles : contrairement aux "grandes montagnes à gruyère » que l'on trouve en Tarentaise voisine et qui impliquent un commerce important de fromages vers l'Italie et la Suisse (Onde, 1942), la Maurienne pratique le système de la «petite montagne " où l'on fabrique dans les alpages l'été et à la fruitière du village l'hiver des fromages de garde de petit gabarit qui sont en grande partie destinés à l'auto-consommation (Bozon, 1986). Aucune fruitière importante n'est mentionnée à Aussois avant les années 1960, qui ne produits donc pas de fromages s'exportant loin (gruyère et beaufort). Les ventes d'animaux dans les foires de la vallée sont modestes en comparaison du flux interne aux exploitations et au stock permanent que représente le cheptel. Le troc entre les familles du village permet de compléter le régime alimentaire (la viande des bestiaux abattus est partagée ou échangée contre d'autres denrées, aussi une famille peut n'abattre qu'un seul bestiau au cours de l'hiver). 
Les solidarités villageoises contribuent à ce faible besoin de monnaie de monnaieéchange (notamment report du besoin de monnaie-échange dans le temps grâce au partage et au troc) et de monnaie-épargne (les familles partagent le mulet, échangent des produits et de la main d'œuvre contre un temps de traction animale, etc.). Mais plus encore, on observe à Aussois l'importance des cadres d'organisation collective, l'étendue de leurs objets, et surtout leur grande permanence. Le cadastre seul démontre une très faible plasticité : du XVIIIème siècle aux années 1960 la proportion de terres arables et près bas n'évolue pas, pas plus que les communaux, alpages et forêts, qui représentent plus des trois-quarts de la surface du village (Cadastres de 1728 et 1927; Rambaud Vincienne 1964). L'usage des communaux est minutieusement règlementé et strictement observé jusqu'aux années 1960 - ce qui est courant dans la vallée pour la gestion des alpages communaux (Digard, 1975 ; Bozon, 1986), mais l'est moins pour les terres arables, privées. Or jusqu'aux années 1960, les assolements des terres arables font l'objet d'une gestion collective à l'échelle du village, qui persiste jusqu'aux années 1960, de même que l'entretien des canaux d'irrigation et leur utilisation (Jail 1969, Bozon 1986). Ces cadres collectifs visent à la bonne répartition des ressource communes, mais plus encore participent à les maintenir - on notera donc que si les terres arables sont privées, le maintien de leur fertilité (par le transfert ou le stock d'azote dans la parcelle, mis en jeu par l'assolement) et de leur production (par l'irrigation) est du domaine du collectif. Les cadres de la vie agro-pastorale aussoyenne, qui régissent les usages des ressources communes et ancrent les solidarités villageoises dans la tradition, augmentent la résistance et la résilience collective des systèmes agraires particuliers dans un cadre non monétarisé, et ce même à mesure que le fiduciaire pénètre plus profondément l'économie de la vallée.

Cependant l'autarcie n'est pas totale, et à l'échelle des systèmes agraires, la substitution ou conversion partielle de stock d'azote (fromages, viandes...) en stock de monnaie via la production animale, est un mécanisme essentiel. Nous avons reconstitué les entrées monétaires d'un système exemple de la période ( $c f$. Tableau 2), qui correspondent à la vente dans les foires de la vallée d'une vache tous les 2-3 ans, de quelques moutons, de vente de beurre et fromages et de lait à la fruitière du village (prix des années 1960 d'après Bozon (1986) et Digard (1975)). Les sorties monétaires, qui incluent l'achat chaque année d'un ou deux porcelets, d'une centaine de kilos de féculent (châtaignes d'Ardèche, pâtes, polenta et riz d'Italie), de sucre et sel et de six hectolitres de vins, représentent la moitié des dépenses annuelles. L'autre moitié des dépenses, outre le paiement des impôts, permet de payer les frais d'écoles, d'acheter les habits et divers outillages. Reste alors un flux net d'entrée monétaire qui en moyenne représente 40000 francs par an et permet d'épargner. Pour les plus aisés, les économies assurent les provisions pour risque, du fait de fortes variabilités des productions en montagne. Elles permettent aussi, tous les 12 ans environ, l'achat du mulet de 100000 francs (1/4 de la marge monétaire dégagée chaque année).

Tableau 2. Estimation des entrées monétaires d'un système agro-pastoral vivrier pour une année type, en franc constant de 1950

\begin{tabular}{|l|l|l|c|c|c|}
\hline Ventes & Unité & Quantité & Prix unitaire & Prix total & $\begin{array}{l}\text { Flux d'azote } \\
(\mathbf{k g})\end{array}$ \\
\hline Vache de réforme & Viande $(\mathrm{kg})$ & 140 & 85 & 11900 & 4,3 \\
\hline Veau & Tête & 1 & 100 & 11500 & 3,6 \\
\hline Agneau & Tête & 1 & 150 & 16500 & 3,3 \\
\hline $\begin{array}{l}\text { Lait (sous forme de } \\
\text { Fromage et beurre) }\end{array}$ & litre & 3000 & 25 & 75000 & 15,9 \\
\hline \multicolumn{2}{|r|}{ Total } & & & $\mathbf{1 1 4 9 0 0}$ & $\mathbf{2 7}$ \\
\hline
\end{tabular}

Cette analyse montre la faible pénétration du système agro-pastoral d'Aussois par la monnaie, et le rôle majeur que joue au contraire les flux d'azote. Le système est gouverné par l'azote, peu disponible et pourtant si essentiel, et la nécessité de son transfert au sein du système. 
Les entrées en fiduciaires lui sont intimement liées (vente des productions animales) et les sorties en fiduciaire dédiées à la reproduction du système agraire (dépenses courantes du ménage, outils, épargne pour la traction animale, survie du ménage en cas d'aléas dans la production). La monnaie permet le transfert de certains flux d'azote au sein du système et en ceci sa reproduction, , l'échange d'azote via les flux alimentaires, voire l'achat d'azote reporté dans le temps via l'épargne, mais ne peut s'y substituer complètement, car le maintien de la fertilité du système agraire est ancrée dans le transfert d'azote des productions animales et dans une gouvernance individuelle et collective non monétarisée. Pour un système agraire particulier, le volume d'azote directement associés au volume monétaire est très faible à l'échelle des flux internes et du stock d'azote, la monnaie a une place marginale dans la gouvernance du système, et le mécanisme de création de richesse est bien le transfert et la multiplication de l'azote

\section{$-5-$ \\ La période 1965- 2013 : un système pastoral spécialisé}

\subsection{Fonctionnement général d'un système pastoral spécialisé exemple}

Nous détaillons ici le fonctionnement, en termes de flux d'azote, d'une exploitation agricole exemple contemporaine de la commune d'Aussois ( $c f$. Figure 5). Cette exploitation, une des plus importantes de la commune, est composée d'une famille de 3 personnes dont une seule travaille sur l'exploitation. La SAU est de 150 ha répartie entre prés bas (50 ha) et prés haut (100 ha), exploités par un cheptel d'une quarantaine de vaches, d'une centaine de brebis et d'une trentaine de chèvres.

Il n'y a pas de terre arable, ni d'animaux de basse-cour pour nourrir la famille. L'objectif principal est la commercialisation ( $99 \%$ de l'azote produit est vendu contre 1\% autoconsommé). En effet, depuis les années 1960, trois facteurs limitants ont été levés i) la force de travail grâce à la mécanisation (tracteur, matériel de fauche, traite mécanique...), ii) l'entretien de la fertilité des sols avec l'apport d'engrais synthétiques (correspondant à $25 \%$ des apports d'azote) et iii) l'importation de céréales pour l'alimentation du bétail (13\% de l'azote des rations animales). Les prés bas fortement fertilisés et productifs apportent toujours l'essentiel de l'azote des rations animales $(65 \%)$, suivis par les prés hauts moins productif $(22 \%)$. Le système a une autonomie de $67 \%$ (soit $4000 \mathrm{~kg} \mathrm{~N} /$ an de fertilisant synthétique et aliments du bétail importés sur un flux total de $12355 \mathrm{~kg} \mathrm{~N} / \mathrm{an})$.

La principale production est le lait pour la fabrication de Beaufort (près de $150000 \mathrm{~L} / \mathrm{an}$ ). Le rendement pour l'ensemble des productions est de $7,2 \mathrm{~kg} \mathrm{~N} / \mathrm{ha} / \mathrm{an}$, soit 2,5 fois plus que le système vivrier exemple d'avant 1965. Les entrées d'azote ont, elles aussi, fortement augmenté ; on les estime à $82 \mathrm{~kg} \mathrm{~N} / \mathrm{ha} / \mathrm{an}$, soit 2,5 fois plus que le système vivrier. Ces chiffres montrent que la production par hectare a fortement augmenté contrairement à l'efficacité de l'utilisation de l'azote qui est de $11 \%$. 
Figure 5. Les flux d'azote en kg/an d'un système pastoral spécialisé exemple (2013)

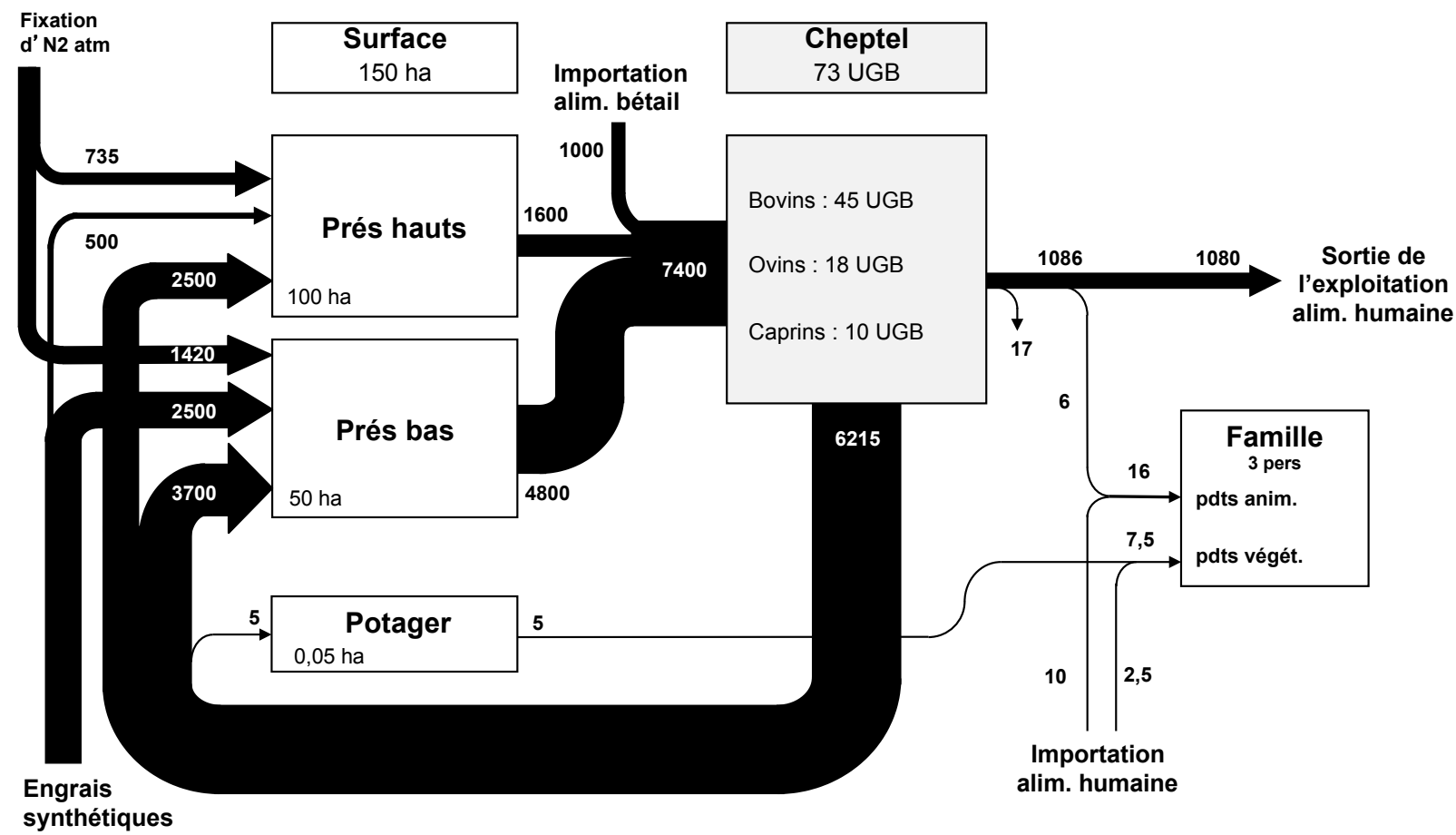

Il est important de souligner que même si les apports d'azote restent limités (pour notre exemple, de l'ordre de $40 \mathrm{~kg} / \mathrm{ha} / \mathrm{an}$ d'azote organique et $20 \mathrm{~kg} / \mathrm{ha} / \mathrm{an}$ de minéral), ils peuvent engendrer des pollutions diffuses des eaux. En effet, cette moyenne sur l'ensemble de la SAU et l'ensemble de l'année tend à gommer la concentration des épandages et déjections sur certaines périodes de l'année et certaines zones. En effet, la période automnale et hivernale sans épandage possible est très longue et les surfaces épandables accessibles sont plus réduites que la SAU totale. Il peut donc exister un problème de stockage et d'épandage de fumier, qui passe du statut de richesse indispensable au maintien du système à celui de problème environnemental à gérer. Le système de production agricole et la consommation du village se sont considérablement élargis et ne sont plus interdépendants comme dans les années 1960.

\subsection{Les flux monétaires d'un système pastoral spécialisé exemple}

Il est important de rappeler ici que nous étudions un système de production certes spécialisé mais extensif comparé aux autres exploitations laitières françaises. Extensif du fait de son utilisation faible d'intrants (aussi bien en ce qui concerne l'alimentation du bétail, que les engrais, le combustible, ...), de ses densités animales faibles, de ses surfaces importantes en prairies naturelles et de la limitation de production de lait par vache (5000 L/an/vache). Extensif, ce système n'en est pas moins formidablement ouvert et pénétré par la monnaie en comparaison de la première période. La productivité du travail a fortement progressé, le nombre de travailleurs étant passé de 6 à 1,2 alors que la production a été multipliée par 17. Une main d'œuvre en 1960 produisait $10 \mathrm{~kg}$ N/an alors qu'elle en produit aujourd'hui près de $900 \mathrm{~kg} \mathrm{~N} / \mathrm{an}$. Pour comprendre le système pastoral spécialisé, il faut désormais adjoindre aux flux d'azote les flux monétaires.

L'agriculture aussoyenne s'est spécialisée dans la production lait pour la production du Beaufort et d'autres fromages de montagne, avec un cahier des charges encadrant les pratiques de production pour garantir la typicité du produit. En contrepartie de la limitation de la production et des intrants, cette production d'appellation d'origine contrôlée (AOC) valorise très bien le lait (voir Buclet aussois pour une analyse plus complète). Le lait est en effet rémunéré de 550 à $660 € / 1000 \mathrm{~L}$, soit de 1,5 à 2 fois plus que la filière conventionnelle. La vente de lait constitue $70 \%$ du produit brut d'exploitation. 
Du fait de sa disponibilité accrue et de son coût très réduit (aussi bien sous forme minérale que dans l'alimentation du bétail), l'azote ne détermine plus la structure et le fonctionnement du système. Le prix de l'azote minéral a été divisé par 100 en un demi-siècle, ceci justifiant en partieque les flux monétaires aujourd'hui ne puissent plus être expliqués par la seule circulation de l'azote au sein du système. La survie du ménage est assurée en plus ou moins grande partie par des revenus extérieurs (la double activité des ménages agricoles est la norme dans la vallée). A l'échelle de l'exploitation, la reproduction et la productivité du système (achats d'intrants et de machines) nécessitent des flux monétaires, comme valeur d'échange et de provision. Plus encore, si le transfert et la multiplication d'azote reste le mécanisme essentiel de création de richesse, il est structuré par les flux monétaires. Les flux monétaires suscitent, orientent et bornent l'ensemble du système en tant qu'ils sont devenus l'outil majeur de la gouvernance du système, notamment pour l'arbitrage de l'allocation des ressources disponibles. La substituabilité entre la monnaie et l'azote est bien plus poussée qu'en première période mais reste imparfaite : le prix de l'azote dans le système varie largement entre l'entrée (prix de la fertilisation azotée et de l'alimentation animale, prix de l'amortissement des investissements) et la sortie (prix du lait), plus encore si l'on ajoute le prix du transfert d'azote en interne (coût de la main d'œuvre et de l'amortissement des machines). Les flux monétaires sont alors des indicateurs pour la gestion du système (Excédent brut d'exploitation) et des signaux forts (prix $\mathrm{du}$ lait ou de l'alimentation animale) pour orienter la gouvernance du système, et s'accompagnent d'indicateurs de gestion ad hoc ( " charges opérationnelles » et " marge brute »). Ces flux n'en restent pas moins orientés et bornés par des cadres plus généraux, d'organisation de marché ou territoriale. Les études de la chambre d'agriculture sur les exploitations spécialisées en « lait Beaufort » de Haute-Maurienne témoignent d'une pénétration monétaire du système pastoral spécialisé largement orientée et bornée par le système d'AOC. Le système, jugé à l'aune de sa productivité monétaire, est soutenu par un prix relativement rémunérateur du lait, mais aussi par des aides publiques agricoles (européennes et nationales, liées à la production et au statut montagnard, qui correspondent à près du quart du produit brut de l'exploitation en 2010), et les contraintes de l'AOC Beaufort sur la fertilisation azotée et l'alimentation animale maintiennent des charges opérationnelles faibles (10 à $15 \%$ du produit brut). La stabilité du prix et des volumes de production du lait relativement stables grâce au cadre de l'AOC limite par ailleurs les risques de production et de marché - cette fonction est généralement assurée par l'épargne, or les systèmes étudiés témoignent d'un endettement relativement faible. Enfin, l'excédent brut d'exploitation moyen des systèmes pastoraux spécialisés de Haute Maurienne est multiplié par deux par rapport au prix du lait non AOC, toutes choses égales par ailleurs. Avec l'AOC Beaufort, les exploitations aussoyennes sont intégrées dans des systèmes d'organisations territoriales et d'activation de ressources communes (Buclet ??) qui, comme les anciens cadres de la vie agro-pastorale, augmentent la résilience et la résistance des systèmes via une gestion des ressources communes (paysages, valeur immatérielle associée aux produits de la montagne) et une certaine autonomie à l'échelle du système d'exploitation. Ainsi la pénétration monétaire des systèmes pastoraux aussoyens, conjointe à leur ouverture et leur spécialisation, n'efface pas poids des transferts non monétarisés d'azote dans le système, et les systèmes de gestion collective des ressources continuent d'assurer la persistance des systèmes pastoraux dans un cadre non monétaire.

\subsection{Fonctionnement général de l'approvisionnement alimentaire à Aussois}

L'activité de la commune d'Aussois, comme celle de toute la vallée de l'Arc, est aujourd'hui centrée sur le tourisme. Le désenclavement de la région et de nouvelles opportunités de travail (construction des infrastructures hydroélectriques, métallurgie, ski...), loin de provoquer un exode rural, ont permis une augmentation de la population communale et conduit à un accroissement considérable de la demande alimentaire liée à l'afflux de touristes (GALLETY, 1983). Là où cinquante ans auparavant, une quasi autonomie alimentaire existait, un 
élargissement considérable de la zone d'approvisionnement du département de la Savoie s'observe aujourd'hui : la Savoie ne subvient qu'à $45 \%$ de ses besoins en protéines, et dépend pour le reste d'importations d'une grande partie du territoire national et de l'Italie (cf. Figure 3 ).

Figure 3. Aire d'approvisionnement alimentaire de la Savoie en termes de protéines : fraction de la demande alimentaire fournie par les départements français en 2006

$\%$ d'origine dans la consommation alimentaire

\section{6}

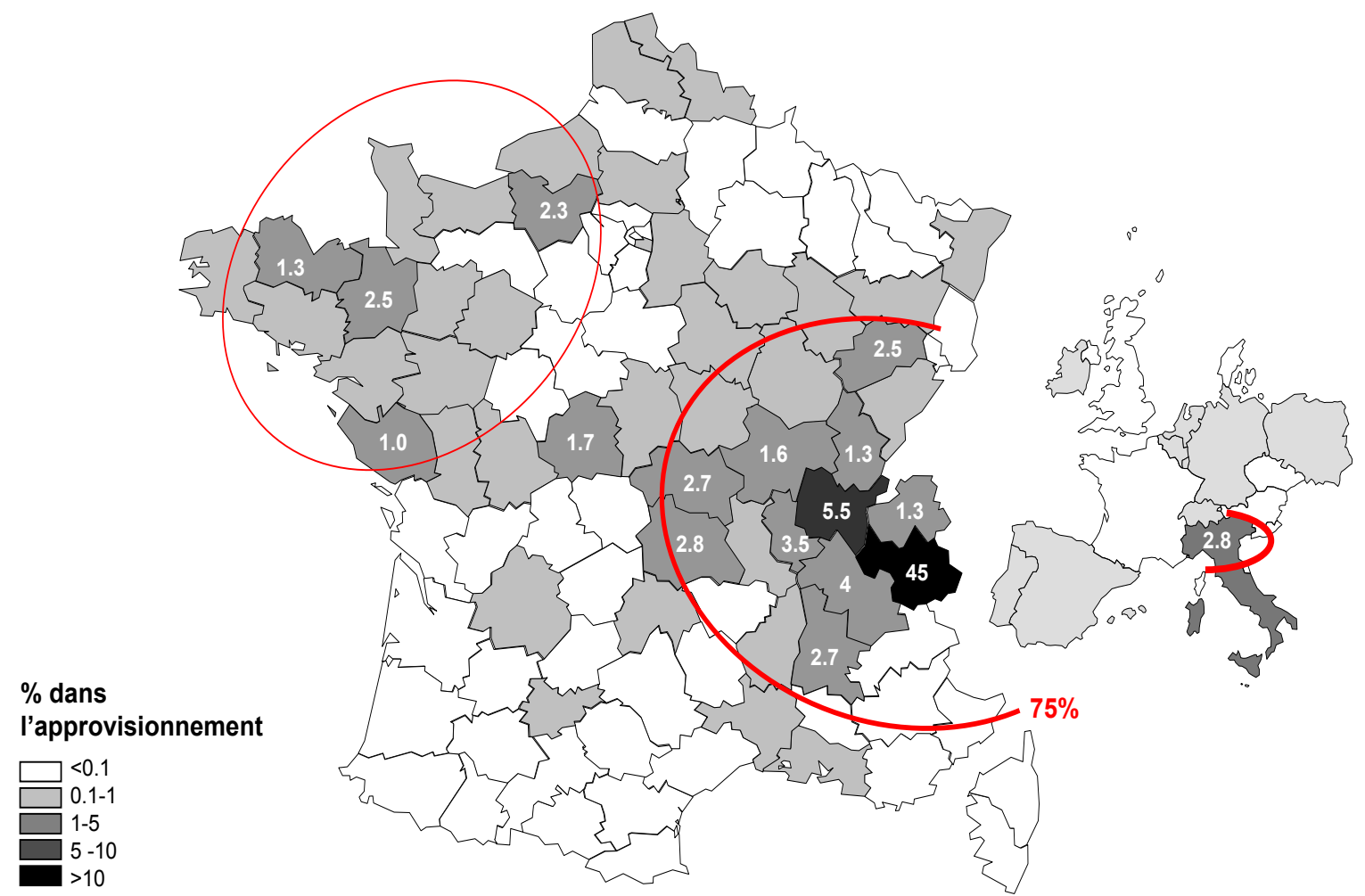

Source: base SITraM des transports de marchandises inter-départementaux, analysée par le logiciel Amstram (SILVESTRE et al., 2015)

Les enquêtes qualitatives montrent que l'approvisionnement alimentaire s'inscrit dans un cadre national, la part de marché assurée par les produits aussoyens est marginale ( $c f$. Figure 4). Comme l'ensemble des français, les Aussoyens s'approvisionnent principalement en grandes surfaces (Casino et Intermarché de Modane et de Sollières-Sardière) et utilisent les supérettes d'Aussois en dépannage (Proxi et Vival). Les grandes surfaces et supérettes ont des stratégies d'approvisionnement locales, régionales et nationales liées à leur groupe respectif. Cependant les approvisionnements proviennent principalement de leur centrale d'achat nationale, seuls quelques produits régionaux sont vendus, mais cela correspond à des volumes minimes (vin, charcuterie, fromages). Les commerces de détail spécialisés (boucherie, fromagerie...) sont principalement utilisés par les populations saisonnières, de plus ces commerces sont eux aussi principalement approvisionnés par des réseaux de distribution classiques (grossistes, marchés de gros). Les marchés en saison touristique sont éventuellement fréquentés par les Aussoyens bien que peu fournis et n'ayant pas une offre différenciée par rapport aux grandes et moyennes surfaces et aux commerces de bouche.

L'analyse de l'offre touristique permet de déterminer que trois-quarts des résidents saisonniers s'alimentent par les mêmes canaux d'approvisionnement que les Aussoyens. Du fait de l'activité touristique la restauration collective et privée participe au moins pour $1 / 4$ au système alimentaire communal. Après enquête, il semble que la part d'approvisionnement 
localisé soit minoritaire (en volumes) du fait d'une faible variété des produits (viande, produits laitiers) (cf. Figure 4).

Figure 4. Modalités et acteurs de l'approvisionnement alimentaire à Aussois

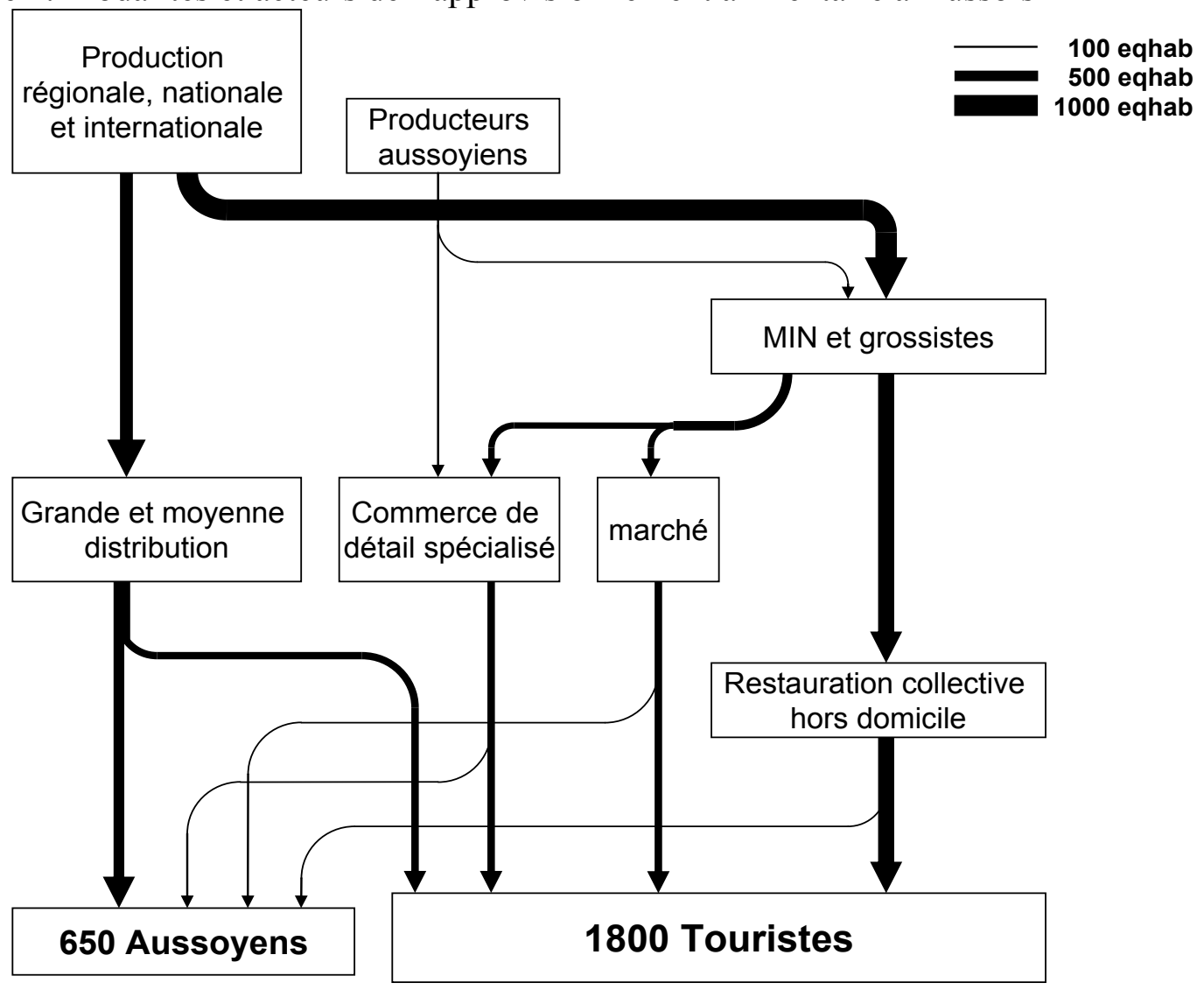

Dans ce contexte économique dominé par le tourisme et une accessibilité élargie aux biens alimentaires, l'activité agricole cesse de jouer un rôle vivrier et son rôle économique à l'échelle de la commune n'est plus essentiel. La production agricole est entièrement recentrée sur l'élevage, avec des productions ancrées dans un territoire et une histoire avec une AOP (Appellation d'Origine Protégée) le Beaufort et des IGP (Indication Géographique Protégée) fromagère Emmental et Tomme de Savoie commercialisées dans l'ensemble de la France. La production laitière est collectée et transformée par "Haute Maurienne-Vanoise », une des trois coopératives de la Maurienne produisant du Beaufort et de la Tomme de Savoie, assurant aux exploitations un débouché commercial et une plus-value importante par rapport au lait standard. La production de viande ovine se fait pour l'essentiel sous le label «Agneau de Sisteron ». Ces signes de qualité (label ou appellation) ont permis le maintien d'une agriculture, qui occupe la majeure partie du territoire. Même si son poids économique direct est relativement faible, l'élevage est essentiel pour l'activité touristique et la pratique du ski par sa fonction d'entretien et d'ouverture du paysage (IDELE, 2006 ; MARINI et al., 2011 ; Mac DonALD et al., 2000 ; MотTET et al., 2006). Au travers des produits certifiés, l'élevage participe aussi à la réputation et à l'image du village auprès des touristes. L'élevage a aujourd'hui une fonction patrimoniale importante pour l'image, l'identité et la vie économique de ce village. 


\section{$-6-$ \\ Discussion et Conclusion}

La mise en perspective des deux périodes par la quantification des cycles de l'azote de deux systèmes exemples montre une ouverture des cycles de matières sur un territoire très vaste et un découplage progressif de la production agricole et de la consommation. Cette dynamique est la résultante d'un désenclavement de la région, d'innovations techniques importantes (azote minéral, mécanisation...) et d'un changement radical du rôle joué par la monnaie.

La monnaie est passée d'un instrument facilitant les échanges agricoles régionaux et l'épargne à un instrument d'échange sur le marché national et international. Jusqu'aux années 1960, la monnaie est intégrée au système agro-alimentaire et ce sont les flux de matières qui pilotent les flux monétaires. Le système financier est encore enchâssé, au sens de POLANYI (1944), dans l'économie, et le transfert et la multiplication d'azote au sein de systèmes agraires peu monétarisés sont le mécanisme essentiel de création de la richesse. L'autonomie du système agricole est un objectif majeur à l'échelle de l'exploitation et du village, et le recours à l'argent ne survient que comme complément à la vie agro-pastorale. Karl Polanyi caractériserait cette société comme héritière des sociétés non marchandes. Aujourd'hui, les flux monétaires, issus de la vente des produits et des politiques publiques agricoles (subventions, crédits, ...), conditionnent entièrement les flux de matières.

Malgré les modifications des systèmes de polyculture-élevage au cours du siècle dernier, cette région de montagne n'a pas atteint le niveau de déconnexion du bassin parisien par exemple (BILLEN et al., 2012) caractérisé par une production céréalière d'exportation basée sur une fertilisation majoritairement minérale ou du bassin d'élevage Breton fortement dépendant aux importations d'intrant comme le soja. Il apparaît toutefois que l'agriculture vivrière où l'animal a une fonction de production alimentaire pour l'autoconsommation, de transfert de fertilité, d'accès au revenu et de capitalisation a été substituée par un système agro-pastoral spécialisé, dominé par une agriculture commerciale où l'animal a un rôle de production alimentaire labellisée (cf. Beaufort, Agneaux de Sisteron). Les engrais minéraux encore peu ou pas utilisés jusque dans les années 1960, représentent désormais $30 \%$ des apports d'azote totaux. Le fumier reste une source locale de fertilité des sols à gérer à partir de bonnes pratiques (BONAUDO et al., 2014). Si les flux monétaires sont largement plus présents dans l'exploitation, ils restent orientés et bornés dans des cadres de gestion collective des ressources territoriales, notamment immatérielles. Ainsi situé, l'élevage sous AOC Beaufort a un rôle d'entretien des paysages et patrimonial support de l'activité touristique (MARINI et al., 2011 ; Mac DoNALD et al., 2000 ; MOTTET et al., 2006).

Le métabolisme territorial par l'inventaire des flux de matières et d'énergie apparaît comme une méthodologie pertinente pour analyser les systèmes agro-alimentaires et leurs trajectoires. En effet, il permet de relier la production et consommation à plusieurs échelles spatiales et temporelles (du système de production, au territoire voire à l'échelle planétaire). L'analyse de flux de matière permet de comprendre la nature et de l'intensité des besoins matériels d'un territoire (par catégorie de ressource et secteur d'activité). Il permet aussi de relier plusieurs activités économiques entre elles à l'échelle du territoire. Nous l'avons appliqué à l'échelle de systèmes de production exemples représentatifs de l'évolution des systèmes agraires du 20 ème siècle de la commune d'Aussois. Pour avoir une vision systémique nous avons quantifié deux types de flux et stocks : l'azote et la monnaie. Cette vision systémique est renforcée par le fait que l'on peut développer des analyses simultanées des utilisations d'intrants (ou moyens de production), des productions végétales et animales (végétaux, viande, lait...), des pertes dissipatives vers l'environnement (pollutions diffuses de l'azote), de l'autonomie du système et de son efficacité de transformation des intrants en produits. Cette analyse simultanée avec plusieurs unités de mesure et plusieurs indicateurs d'efficience est essentielle aujourd'hui pour repenser le fonctionnement des systèmes d'élevage, leurs articulations en filière et au territoire. 
Pour maintenir un niveau élevé de production tout en diminuant les impacts environnementaux, il est essentiel de comprendre finement les interactions entre activités d'élevage et flux de matière à l'échelle des exploitations et des territoires. En complément de cette analyse biophysiques, des études des réseaux d'acteurs partie prenante du système agro-alimentaire pourraient être menées. En revanche, cette méthode basée sur l'étude de flux ne permet pas d'analyser des fonctions essentielles de l'élevage comme l'entretien du paysage, la valeur patrimoniale, esthétique, culturelle ou identitaire. Pour cela une analyse systémique de l'élevage dans un territoire devra être faite avec plusieurs disciplines aussi bien biotechnique (écologie, agronomie, écologie territoriale, ...), que de sciences économiques et sociales (analyse économique, analyse de réseau d'acteurs et de gouvernance, de verrouillage sociotechnique...).

\section{Références bibliographiques}

Agreste (2011) Une Savoie Toujours en Herbe. Rhône-Alpes Coup d'oeil n 130 - novembre 2011- Recensement agricole 2010. http://www.agreste.agriculture.gouv.fr/IMG/pdf_D7311A01-2.pdf

ANGLAdE J, BILlEN G, GARNIER J (2015) Relationships for estimating N2 fixation in legumes : incidence for N balance of legume-based cropping systems in Europe, Ecosphere, 6(3), art 37, 24 p.

http://onlinelibrary.wiley.com/store/10.1890/ES14-

00353.1/asset/ecs214003531.pdf;jsessionid=A0A172DDB569B0AB018EF93E4B639107.f04t02? $\mathrm{v}=1 \& \mathrm{t}=\mathrm{ith} 4 \mathrm{~m} 4 \mathrm{zq}$ $\& \mathrm{~s}=\mathrm{aab} 3 \mathrm{df5}$ fecf2cd4fc3f076fafe1ae0d5940b2cde

BARLES S (2010) Ecologie urbaine, industrielle et territoriale, in Coutard O, Levy J-P (dir.), Écologies urbaines. Economica, pp. 61-83. Paris.

Billen G, GARnier J, Thieu V, Silvestre M, BARles S, Chatzimpiros P (2012) Localising the nitrogen imprint of Paris food supply: the potential of organic farming and changes in human diet. Biogeoscience, 9, 607-616.

BILlen G, LASSAlEtTA L, GARNIER J (2014) A biogeochemical view of the global agro-food system: nitrogen flows associated with protein production, consumption and trade. Global Food Security, 3 (3-4), $209-219$.

BiLlen G, LASSALETTA L, GARNIER J (2015) A vast range of opportunities for feeding the world in 2050 : trade-off between diet, N contamination and international trade. Environ. Res. Lett., 10(2), 15p.

Bodirsky BL, Popp A, Weindl I, Dietrich JP, Rolinski S, Scheiffele L, Schmitz C, Lotze-CAmpen H (2012) $\mathrm{N}_{2} \mathrm{O}$ emissions from the global agricultural nitrogen cycle - current state and future scenarios. Biogeosciences, 9 (4), 169-97.

Bonaudo T, Bendahan AB, Sabatier R, Ryschawy J, Bellon S, Leger F, Magda D, Tichit M (2014) Agroecological principles for the redesign of integrated crop-livestock systems. Eur. J. Agron., 57, 43-51.

Bourgeois L, DEMOTES-MAINARD M, (2000) Les cinquante ans qui ont changé l'agriculture française. Économie rurale, 255(1), 14-20.

Bulletin de la Société française d'économie rurale (1951) L'utilisation des tracteurs dans les exploitations. Volume $3 \mathrm{~N}^{\circ} 1,1951$. L'utilisation des tracteurs agricoles dans quelques régions de la France (résultats d'une enquête dans 500 exploitations agricoles). pp.13-30.

Cas types bovin lait Rhône-Alpes, rapport interne, Pôles d'Expérimentation et de Progrès (2013), consulté le 2 septembre 2014. 6 p. http://www.pep.chambagri.fr/index.php/bovinslait-acces-aux-ressources/economie/452-castype-bovin-lait/427-cas-types-bovin-lait-rhone-alpes

Chambre D’agriculture de Savoie Et De Haute Savoie 2011 : Le Plan Stratégique Agricole et de

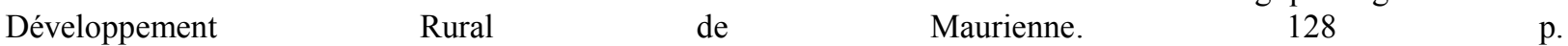
http://www.maurienne.fr/infolivedocuments/pdf/psader_maurienne etat-des-lieux.pdf

CORPEN (1999) Estimation des flux d'azote, de phosphore et de potassium associés aux vaches laitières et à leur système fourrager. http://preprod.meeddm.nexint.net/IMG/pdf/DGALN_1999_11_flux_laitiere_fourrager.pdf CORPEN (2001) Estimation des flux d'azote, de phosphore et de potassium associés aux bovins allaitants et aux bovins en croissance, issus des troupeaux allaitants et laitiers, et à leur système fourrager. http://www.developpementdurable.gouv.fr/IMG/pdf/DGALN_2001_06 flux bovin_engrais.pdf

Des systèmes d'exploitation à dominante laitière pratiqués en Rhône-Alpes et PACA : en haute montagne alpine : un système spécialisé traditionnel producteur de lait à Beaufort, rapport interne, Pôles d'Expérimentation et de Progrès (2004) consulté le 2 septembre 2014 p6.

http://www.pep.chambagri.fr/mydms/pep_bovins_lait/file_4e708049df422.pdf.

FISCHER-KOWALSKI M, HABERL H (eds) (2007) Socioecological transitions and global change. Trajectories of social metabolism and land use. Cheltenham : Edward Edgar.

GALLETY JC (1983) Les sociétés montagnardes devant l'aménagement touristique : l'émergence du fait communal, thèse de 3e cycle, Institut d'urbanisme, Université des Sciences sociales de Grenoble, 600 p. 
GARDELLE F (1965) La transhumance ovine entre les régions méditerranéennes et les Alpes en 1964. Revue de géographie alpine, 53 (3), 449-477.

GARDELLE F ET GARDELLE C (1976) Histoire d'un alpage communautaire, l'alpage de Loriaz (Haute-Savoie). Revue de géographie alpine, 64(2), 185-205.

GUERIN JP (1972) Les fruitières savoyardes (Savoie et Haute-Savoie). Revue de géographie alpine, 60(3), $453-466$. IDELE (2010) Institut de l'Elevage, Réseau d'élevage, Des systèmes d'exploitation à dominante laitière pratiqués en Rhône Alpes et PACA, En haute montagne alpine, un système spécialisé traditionnel producteur de lait à Beaufort. http://www.pep.chambagri.fr/mydms/pepbovins lait/file 4e708049df422.pdf.

IDELE (2006) Paysages d'élevages - Paysages d'éleveurs. Collection synthèse. 40p.

INAO (2010) Cahier des charges de l'appellation d'origine « Beaufort », version no 14 du 8/11/2010.

INSEE (2011) Population légale d'Aussois en 2011. http://www.insee.fr/fr/ppp/bases-dedonnees/recensement/populations-legales/commune.asp?depcom=73023

JAIL M (1969) La Haute-Maurienne. Recherche sur l'évolution et les problèmes d'une cellule montagnarde intraalpine. Revue de géographie alpine, 57(1), 85-146.

Lassaletta L, Billen G, GRIZZETti B, Anglade J (2014a) 50 year trends in nitrogen use ef fi ciency of world cropping systems : the relationship between yield and nitrogen input to cropland. Environ. Res. Lett. 105011.

Lassaletta L, Billen G, Grizzetti B, Anglade J, Garnier J (2014b) Supplementary Information to the paper (Suppl. 1) 50 year trends in nitrogen use efficiency of world cropping systems : the relationship between yield and nitrogen input to cropland. Environ. Res. Lett. 1-12.

Le revenu de l'agriculture en 1950-51, in Etudes et conjoncture - Union française / Economie française, 6e année, no 5, 1951, 38-61.

Mac Donald D, Crabtree JR, Wiesinger G, Dax T, Stamou N, Fleury P, Gutierrez LazPita J, Gibon A, (2000) Agricultural abandonment in mountain areas of Europe: Environmental consequences and policy response. Journal of Environmental Management. 59(1), 47-69.

MALASSIS L (1994) Nourrir les hommes. Flammarion, Paris, 126 p.

MARINI L, KLIMEK S, BATTISTI A (2011) Mitigating the impacts of the decline of traditional farming on mountain landscapes and biodiversity: a case study in the European Alps. Environmental Science \& Policy, 14(3), $258-267$.

MARNEZY A (1979) Les stations de ski de la Haute-Maurienne : un exemple original d'aménagement touristique. In : Revue de Géographie Alpine, tome 67 n³, 281-307.

MAZOYer M, Roudart L (2002) Histoire des agricultures du monde. Du néolithique à la crise contemporaine. Editions du Seuil, Paris, 736 p.

Mottet A, Ladet S, Coqué N, GiBon A (2006) Agricultural land-use change and its drivers in mountain landscapes: A case study in the Pyrenees Agriculture. Ecosystems \& Environment, 114 (2-4), 296-310.

Observatoire des territoires de la Savoie Etat des lieux agriculture, urbanisme et territoire Maurienne http://www.observatoire.savoie.equipement-agriculture.gouv.fr/PDF/Comment/Etat lieu_Maurienne.pdf

ONDE H (1937) Jachère climatique et servitudes agricoles en Haute-Maurienne, in Annales de Géographie, t. 46, no 262. p. 369-373.

POLANYI K (1944) The Great Transformation: the political and economic origins of our times. Beacon Press, Boston.

RAMBAUD P et VINCIENNE M (1964) Les transformations d'une société rurale: La Maurienne 1561-1962, Armand Colin, Paris.

Silvestre M, BILlEN G, GARNIER J (2015) Évaluation de la provenance des marchandises consommées par un territoire, PP 361-370. $1^{\mathrm{er}}$ colloque interdisciplinaire sur l'écologie industrielle et territoriale, COLEIT 2012. Junqua G. et Brullot S. coord. Presse des Mines, Alès.

VIALLET H (1993) Les alpages et la vie d'une communauté montagnarde: Beaufort du Moyen Âge au XVIIIe siècle, Mémoire publié par l'Académie Salésienne, t. 99, n¹5, Gallica. Consulté le 24 juin 2014. URL: http://gallica.bnf.fr/ark:/12148/bpt6k5834342c/f69.image.

Arbos 1919), «La communication dans les Alpes françaises », Annales de géographie, t. 28, $\mathrm{n}^{\circ} 153$, pp. 161-176

Chamussy 1968, «Circulation transalpine et ville de pied de col. Briançon, Modane, Suse, Aoste, Martigny, Brigue, Domodossola », RGA, tome 56, n³-4, pp. 425-468).

Gardelle 1965, "La transhumance ovine entre les régions méditerranéennes et les Alpes en 1964 ", RGA, t. 53, n³, pp. 449-477. 